\title{
Verapamil Ameliorates the Clinical and Pathological Course of Murine Myocarditis
}

Raymond Dong, Peter Liu, Lily Wee, Jack Butany, and Michael J. Sole

Centre for Cardiovascular Research, Division of Cardiology, Department of Medicine, and Department of Pathology, The Toronto Hospital, University of Toronto, Ontario, Canada M5G 2C4

\begin{abstract}
The effects of the calcium channel blocking agent, verapamil, were studied in a murine model of viral myocarditis. Three groups of 8-wk-old DBA / 2 mice $(n=25$ each) were inoculated with 10 plaque-forming units of encephalomyocarditis virus and randomized to three treatment regimens. Group 1 mice received verapamil intraperitoneally $(5 \mathrm{mg} / \mathrm{kg}$ per d) for $7 \mathrm{~d}$ before infection, followed by verapamil orally (mean dose of 3.5 $\mathrm{mg}$ (mouse per d) in drinking water during infection. Group 2 mice received only verapamil orally starting on day 4 after infection, coincident with peak viremia. Group 3 (infected control) received no verapamil in regular drinking water after viral inoculation. Additional control animals were studied in group 4 $(n=21)$, consisting of uninfected control animals receiving intraperitoneal and oral verapamil at doses identical to group 1 , and in group $5(n=21)$, consisting of uninfected and untreated controls. Animals were randomly killed from each group ( $n$ = 7) at 7, 14, and $28 \mathrm{~d}$ after infection. Routine histology was performed blindly on an apical slice of each heart and semiquantitatively graded for inflammation, necrosis, calcification, and fibrosis on a scale of $0-4$. Digital planimetry was performed to measure the absolute and relative areas of inflammation and necrosis. The pretreated animals in group 1 showed marked reduction in inflammation and necrosis (score of $3.7 \pm 1.4$ vs. $8.7 \pm 2.0$ in group 3 on day $14, P<0.05$ ) and were indistinguishable from the posttreated group 2 mice (score of 4.0 \pm 1.5 vs. $8.7 \pm 2.0$ in group 3 on day $14, P<0.05$ ). All the uninfected control animals (groups 4 and 5 ) showed no myocardial lesions whether treated with verapamil or not. Quantitative planimetry confirmed decreased inflammation and necrosis (2.0 $\pm 3.3 \%$ in group 1 and $3.5 \pm 3.1 \%$ in group 2 vs. $21.9 \pm 22.6 \%$ in group 3 on day 14). Untreated infected hearts injected with liquid silicone rubber exhibited extensive areas of focal microvascular constriction and microaneurysm formation; verapamil treatment in either group 1 or 2 completely abolished these abnormalities, resembling uninfected controls in groups 4 or 5 . We conclude that verapamil, whether given before infection or after peak viremia in an encephalomyocarditis model of murine myocarditis, significantly reduces the microvascular changes and myocardial necrosis, fibrosis, and calcification leading to
\end{abstract}

Presented in part at the American Federation of Clinical Research National Meeting, Seattle, WA, 1991.

Address correspondence to Dr. Peter Liu, Director, Cardiology Research, GW 1-512, Toronto General Hospital, Toronto, Ontario, Canada, M5G 2 C4. 1992.

Received for publication 11 June 1991 and in revised form 7 May

J. Clin. Invest.

(C) The American Society for Clinical Investigation, Inc.

0021-9738/92/11/2022/09 \$2.00

Volume 90, November 1992, 2022-2030 cardiomyopathy. This suggests the potentially important role of calcium and microvascular spasm in the pathogenesis of viral myocarditis leading to dilated cardiomyopathy, and may have future therapeutic implications. (J. Clin. Invest. 1992. 90:2022-2030) Key words: calcium channel blocker • myocardial inflammation - dilated cardiomyopathy - microvascular spasm

\section{Introduction}

Significant evidence has accumulated from both animal models and clinical studies to indicate that there is a close etiologic link between viral myocarditis and idiopathic dilated cardiomyopathy $(1,2)$. A suggested mechanism is the activation of the immune system after viral infection that leads to continued myocyte destruction (3-5). It follows that therapy for viral myocarditis targeted toward the immune system should result in decreased dilated cardiomyopathy. Unfortunately, therapeutic results have been disappointing in both the clinical realm (6, 7 ) and in basic animal paradigms of viral myocarditis. Major therapeutic approaches including immunosuppressive regimens $(8,9)$ and nonsteroidal anti-inflammatory agents $(10$, 11) have all led to conflicting and disappointing results. To show benefit in animal models, such as the encephalomyocarditis (EMC) ${ }^{1}$ infection in DBA/2 mice (12), or Coxsackievirus infection in $\mathrm{BALB} / \mathrm{c}$ mice $(3,13)$, pretreatment is often necessary. Modalities such as prednisolone (14) and cyclosporine (15) have paradoxically exacerbated the disease in these models. Therefore, new therapeutic approaches toward myocarditis appear to be indicated.

Myocarditis is a multifaceted process involving viral infection $(16,17)$, immune activation $(4,18)$, and microvascular spasm (19). Many of the processes that perpetuate the disease are calcium dependent. Verapamil, a slow calcium channel blocking agent, has been shown previously to be effective in ameliorating the clinical and pathologic manifestations of cardiomyopathies, including the genetic Syrian hamster model (20) and the acquired murine Chagasic model (21). Despite their widely differing etiologies, these disorders possibly have in common a disturbance of the coronary microcirculation as the final common pathway toward development of cardiomyopathy. Recently, evidence of similar coronary microvascular spasm was also found in a model of acute murine myocarditis (19). The possibility that calcium channel blockers may play a therapeutic role in altering the natural history of myocarditis leading to cardiomyopathy is an intriguing hypothesis that remains to be tested.

The purpose of this study was to examine the effects of both pre- and postinfection treatment with verapamil on: $(a)$ the pathologic evolution of myocarditis toward cardiomyopathy, and $(b)$ the status of the coronary microcirculation, as com-

1. Abbreviation used in this paper: EMC, encephalomyocarditis. 
pared with untreated infected controls, in an established EMC model of murine myocarditis.

\section{Methods}

\section{Preparation of animal model}

EMC virus ( $M$ variant) was propagated in confluent monolayers of hamster kidney cells (BHK-21 line) using minimal essential media containing DNA, RNA, antibiotics, $40 \mathrm{mM}$ Hepes, and 10\% FCS. After complete cytopathic effect was seen, the supernatant, which contained the virus, was collected after centrifugation at 3,000 rpm and maintained at $-70^{\circ} \mathrm{C}$ until use. Viral titers were determined by plaqueforming assay using the BHK-21 cell line. 8-wk-old male DBA/2 mice (Charles River Breeding Laboratories, Inc., Wilmington, MA) were inoculated on day 0 intraperitoneally with $0.1 \mathrm{ml}$ of virus suspension containing 10 plaque-forming units. The animals were placed in isolated cages and fed with a normal diet and water. The animals were weighed twice weekly and observed for evidence of heart failure, including changes in fur characteristics, level of activity, paw edema, and anasarca. Neurologic sequelae were observed in terms of spastic diplegia and monoplegia. The entire experimental protocol was approved by the University Committee on Animal Care and conducted in accordance with the principles of animal care set out by the American Physiological Society.

\section{Treatment protocol}

After viral inoculation, the animals were randomly allocated to five groups. 25 mice were pretreated with intraperitoneal verapamil (Knoll Pharmaceuticals, Markham, Ontario, Canada) at a dose of $5 \mathrm{mg} / \mathrm{kg}$ per $\mathrm{d}$ for $7 \mathrm{~d}$ before virus inoculation (group 1). The intraperitoneal route was chosen to ensure that a proper dose of verapamil was received by all the animals before viral inoculation, and to maximize any benefit that verapamil may have. These mice continued to receive oral verapamil (dissolved in $10 \%$ dextrose solution at a concentration of 1 $\mathrm{mg} / \mathrm{ml}$ ) immediately after infection, consuming an average dose of 3.5 $\mathrm{mg} /$ mouse per $\mathrm{d}$. A second group of 25 mice received verapamil only in their drinking water $(1 \mathrm{mg} / \mathrm{ml})$, at the same average daily dose, starting after day 4 after viral inoculation (group 2). This coincided with peak viremia in this model and the beginning of observable clinical illness. A third group of 25 mice received no therapy before or after viral inoculation in their drinking water and constituted the infected control group (group 3). A fourth group of 21 mice received intraperitoneal verapamil before inoculation with culture medium that contained no virus. This was followed by a full course of oral verapamil at identical doses to group 1, and constituted the uninfected, treated group (group 4). A final group of 21 mice also received culture medium inoculation without active virus, but received no verapamil treatment at any time, and constituted the uninfected untreated control group (group 5).

Animals from each group were randomly assigned to be killed at 7 , 14 , and $28 \mathrm{~d}$ after infection to determine microvascular and pathological changes at each stage of infection. The days were chosen to coincide with the stages of initial myocardial inflammation ( $7 \mathrm{~d})$, peak mononuclear cell infiltrate ( $14 \mathrm{~d})$, and the beginning of fibrosis and dilated cardiomyopathy ( $28 \mathrm{~d})$.

\section{Pathologic examination}

Microvascular perfusion studies. Immediately before killing, all the animals underwent microvascular perfusion studies. Under sodium pentobarbital anaesthesia, a midline sternotomy was performed on each animal and the left atrium was exposed. Each beating heart was perfused by direct hand injection into the left atrium with $1.0 \mathrm{ml}$ liquid silicone rubber freshly prepared from constituent reagents (Microfil; Canton Bio-Medical Products, Inc., Boulder, CO). After cessation of cardiac contractions, the hearts were rapidly excised and fixed in $10 \%$ formalin overnight at room temperature. The next day, the apical portion incorporating the right and left ventricles was excised for processing and light microscopic examination. The remainder of the heart tissue was sliced in 2-3-mm-thick transverse cross-sections and cleared by sequential $24-\mathrm{h}$ immersions in $25,50,75,95$, and finally $100 \%$ ethyl alcohol. On day 6 , specimens were placed in pure methyl salicylate for 12-24 $\mathrm{h}$. The myocardial tissues became translucent and devoid of all cellular detail after this clearing process. The coronary microcirculation was then visualized with both epi- and trans-illumination. Microfil-perfused vessels were readily examined under low power magnification $(\times 40-100)$.

Light microscopy. Cardiac tissues from the apically excised portions of both ventricles were embedded in paraffin, sectioned at $3 \mu \mathrm{m}$ thickness, and stained with hematoxylin and eosin. Fibrosis and collagen deposition were detected with Masson's trichrome stain. Four to six sections of each heart were scored blindly by a cardiac pathologist (J. Butany). For each myocardial sample, histologic evidence of myocarditis and inflammation was classified in terms of degree of cellular infiltration, myocardial cell necrosis, extent of calcification, and fibrosis and was graded on a five-point scale ranging from 0 to $4+$ (12). A zero score indicated no or questionable presence of lesions in each category. A $1+$ score described a limited focal distribution of myocardial lesions. Scores of $2+$ to $3+$ described intermediate severity with multiple lesions, whereas a 4+ score described the presence of coalescent and extensive lesions over the entire examined heart tissue. A maximum score of 16 was possible using this grading system.

Digital planimetry. A whole-mounted transverse cross-section of each heart was photographed on color slide film and projected onto a computerized digitization tablet. Total myocardial cross-sectional area and absolute area of myocardium involved with inflammation were planimetered using the Bioquant System IV (R \& M Biometrics, Boston, MA), a software package designed for quantitative morphometric analysis. To correct for individual variation in heart size, a percentage of absolute area involved to total myocardial area was also calculated. The degree of pathologic involvement for each individual section was thus expressed both as an absolute area, as well as a relative percentage.

Statistical analysis. Results are presented as group means \pm SD. Comparison within and between treatment groups was performed using analysis of variance (SAS statistical systems). Post hoc comparison of individual groups was done using Newman-Keul subgroup analysis. A $P$ value of $<0.05$ was considered significant.

\section{Results}

Morbidity and mortality. Four animals died in each of groups 1,2 , and 3 , resulting in a mortality of $16 \%$ for verapamiltreated and untreated infected animals. Although mortality rates were similar in each group, the time of death was significantly delayed in the verapamil-treated mice. Group 1 (pretreated) had two deaths on day 12 and two on day 16 . Group 2 (posttreated) similarly had three deaths on day 11 and one death on day 14. Group 3 (infected control) had two deaths on day 5 after infection and two on day 7. Group 4 (uninfected treated) and group 5 (uninfected untreated) incurred no deaths.

Subjectively, the verapamil-treated infected animals were more active throughout the course of the infection. However, the amount of water consumed in each group was comparable. Neurologic sequelae were also attenuated in groups 1 and 2; only 5 and 6 animals of 25 , in groups 1 and 2 , respectively, developed spastic paralysis, compared with 10 of 25 untreated infected mice in group $3(P<0.05)$. The group 4 animals were normally active throughout the study, despite treatment with 


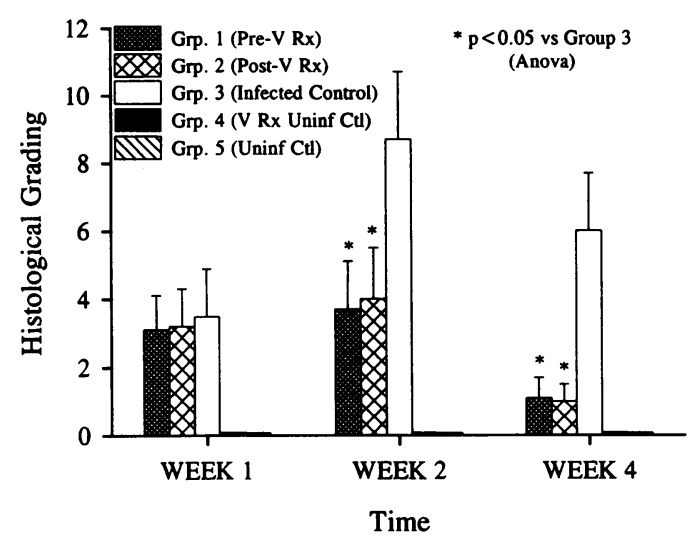

Figure 1. Histological grading according to experimental group and time. Histological scoring of hearts ranged from 0 to $4+$ in each of the categories of inflammation, necrosis, calcification, and fibrosis. Maximum cumulative score was 16 using this method. Data were displayed as group mean $\pm \mathrm{SD}$. $V$, verapamil; $R x$, treatment; $C t l$, control; Uninf, uninfected; Anova, analysis of variance.

verapamil. The activities between groups 4 and 5 were identical.

Histologic examination. Pathologic scores for each group, at 7, 14, and $28 \mathrm{~d}$ after viral inoculation are shown in Fig. 1. As shown in Fig. 2 involving the infected animals, treatment with verapamil markedly attenuated the severity and number of myocardial lesions in these infected animals. There was no significant difference among all three infected groups (groups 1,2 , and 3 ) during the first $7 \mathrm{~d}$. All showed the early presence of mononuclear cell infiltrates in several foci in the heart. By the second week of infection, the untreated infected animals progressed to more extensive myocardial cell necrosis and more widespread inflammatory cell infiltrates. These findings persisted in the untreated infected group (group 3 ) to the end of 28 $\mathrm{d}$ of infection.

On the other hand, most of the hearts in groups 1 and 2 showed significant reversal of the inflammatory and necrotic processes, with marked diminution in myocarditis at $14 \mathrm{~d}$ and very little residual evidence of disease by $28 \mathrm{~d}$ (Fig. 3). In contrast, the group 3 hearts continued to show evidence of myocyte damage with dystrophic calcification and early fibrosis progressing toward the development of a dilated cardiomyopathy. There was a significantly decreased to complete absence of fibrosis in the treated hearts. There was no significant difference in the protective effect of verapamil when administered $7 \mathrm{~d}$ before viral infection or after peak viremia had occurred on day 4 .

Both of the uninfected control groups (groups 4 and 5) showed no myocardial lesions (grade 0 pathological changes) at any time, whether treated with verapamil or not (Fig. 4). This confirmed that verapamil specifically attenuated the myocarditic lesions induced by viral inoculation, and did not have a competing myopathic effect. Small epicardial lesions, however, continued to be seen, predominantly over the right ventricle, but with occasional patches extending over to the left ventricle. These were similar in the control and study animals, and were comprised of inflammatory cells, macrophages, and fibrocalcific foci, as described previously by other investigators (13).

The planimetric measurements of the absolute cross-sec- tional area of myocardial inflammation and necrosis due to the myocarditic process, as well as the affected area expressed as a percentage of total myocardial area are shown in Fig. 5. Again, a dramatic decrease in disease severity was demonstrated in the verapamil-treated infected animals (groups 1 and 2) compared with group 3 untreated infected animals. The uninfected animals showed essentially no myocardial change apart from that seen with normal growth.

Microvascular perfusion studies. The cardiac microvasculature in all untreated infected mice with myocarditis during days 7,14 , and 28 was markedly abnormal. Extensive areas of focal microvascular lumenal narrowing were seen (Fig. $6 a$ ). The contour of these vessels were irregular with focal filling defects and abrupt terminations of these vessels. Numerous areas of pronounced constriction consistent with spasm were found in association with pre- and poststenotic dilatation, giving the appearance of microaneurysm formation. There was a generalized paucity of perfused vessels in the group 3 infected untreated hearts. In contrast, in verapamil-treated infected mice (groups 1 and 2) all hearts contained microvasculature that was smoothly tapered throughout (Fig. $6 b$ ). There was a much greater density of small vessels and no focal narrowings were detected. These findings were almost identical to the uninfected control hearts seen in groups 4 and 5 . The treatment of verapamil in the uninfected hearts appeared to have no effect on the microvasculature.

\section{Discussion}

This study provides the first direct evidence that the calcium channel blocking agent, verapamil, when administered to a well-established EMC DBA./2 murine model of myocarditis, markedly attenuated not only the severity of the myocarditic process but also altered the clinical course of the disease. Moreover, these salutary effects occurred whether the verapamil was given before or after peak viral replication and the onset of definable illness. This suggests that verapamil interfered with the mechanisms that perpetuate the inflammatory myocyte destruction. There are several possible explanations for this substantial improvement.

Our current understanding of myocarditis suggests that it is a three-stage process. There is an initial viral infection of genetically susceptible host myocytes that can lead to the early pathologic evidence of myocardial damage (22). The infectious process itself may alter the myocyte and modulate the immune system in such a manner that cell-mediated immunity activates the second phase of myocardial and endothelial damage $(18,23,24)$. Finally, a chronic phase is characterized by slow myocyte loss and myocardial fibrosis which progresses to a dilated cardiomyopathy. Part of the latter process appears to be perpetuated by persistence of viral genomic material within the affected myocytes as demonstrated by molecular hybridization techniques $(17,25,26)$ and the presence of microvascular spasm (19).

Verapamil has the potential of interfering predominantly with the second and third phases of myocarditis, as it appears to be effective even after the first phase has been completed. However, the possibility that verapamil may attenuate viral infectivity during the initial myocarditic process cannot be completed ruled out, even though this was not a primary goal of this study. 

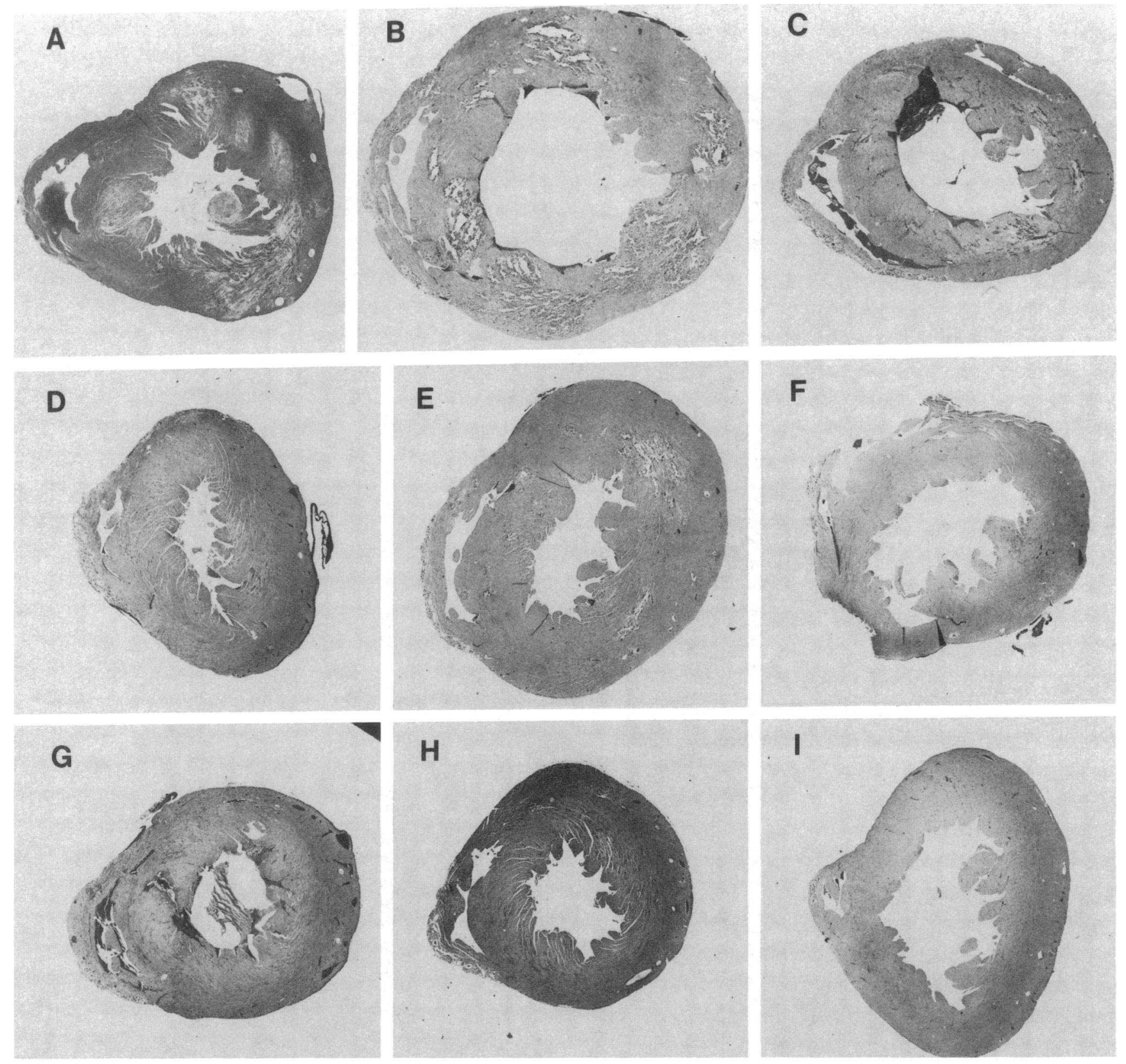

Figure 2. Transverse cross-sectional slices of representative hearts from the infected groups 1, 2, and 3. $A-C$ are from group 3 (control) mice at 7 , 14 , and $28 \mathrm{~d}$, respectively. $D-F$ are from group 1 (pretreated) at similar times. $G-I$ are from group 2 (posttreated). There were significant histological differences noted between groups 1 and 3 and groups 2 and 3, but not between groups 1 and 2 . H \& E staining. $\times 15$.

Certainly, verapamil appears to block replication of certain viruses during the final stage of assembly and viral budding by inhibiting portions of this calcium-dependent process. Decreased intracellular calcium may affect membrane fusion events as well as cytoskeletal organization (27). These events may lead to a lower viral infective burden which in turn may result in lesser amounts of viral genome persisting in host myocytes. Recent studies suggest that the duration and degree of genomic persistence is important in determining the severity of myocarditis (28).

In relation to the second phase of myocarditis, which is characterized by immune-mediated myocyte destruction, the importance of $\mathrm{T}$ lymphocytes has been well demonstrated in several animal models $(4,18,24,29)$. Specifically, the cytotoxic $T$ lymphocyte subset has been shown to target viral-infected cells for destruction (30). However, T cell activation and proliferation are felt to be calcium-dependent processes $(31,32)$. The effect of calcium channel blockers on lymphocyte function has been studied in detail in vitro (32-34), and they were found to have considerable suppressive effects on many aspects of $T$ cell function. For example, the slow and sustained elevation of intracellular free calcium concentration is an essential signal for IL-2 production by $\mathrm{T}$ cells (35). IL-2 itself plays a vital role in perpetuating the $T$ cell immune re- 

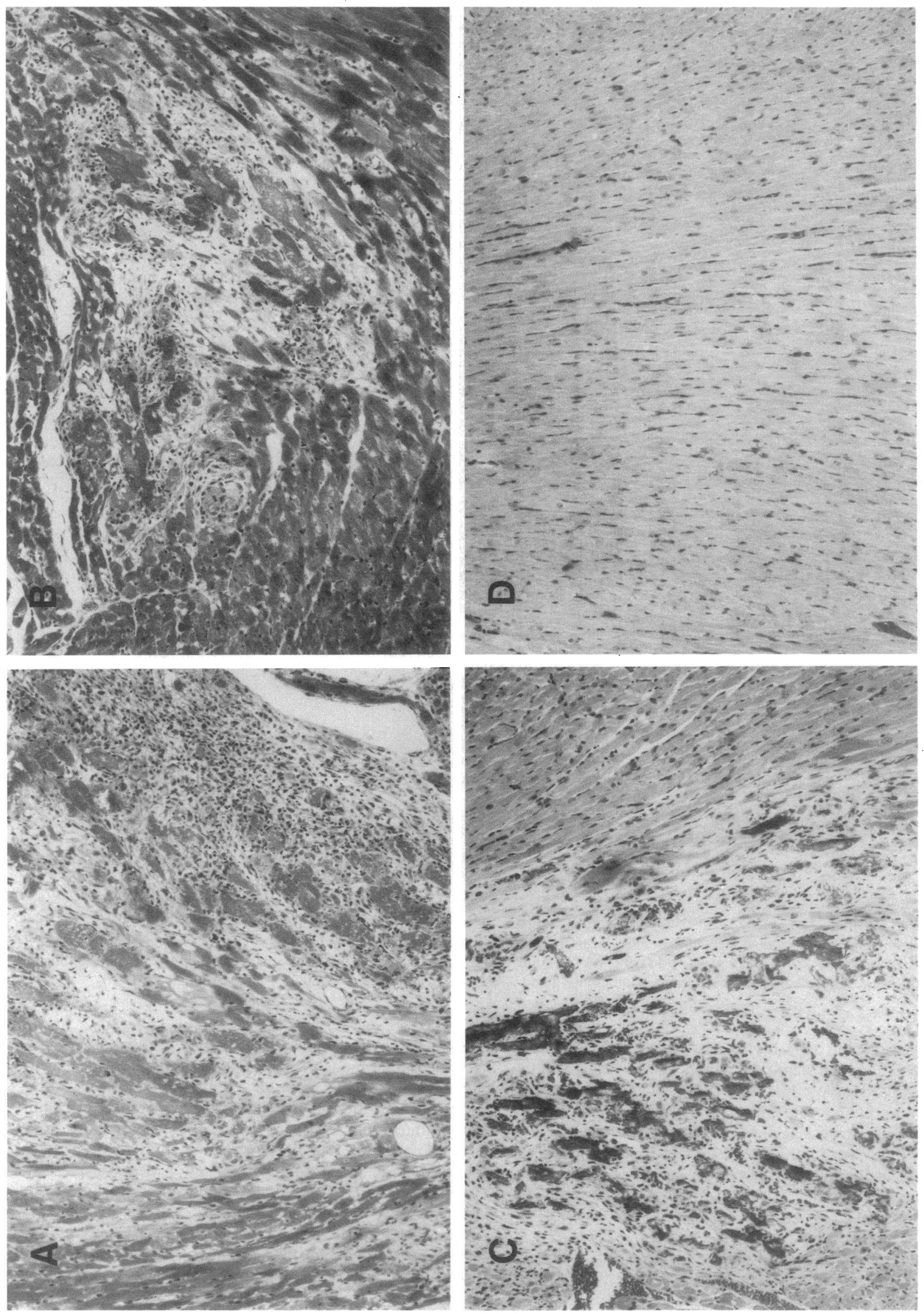


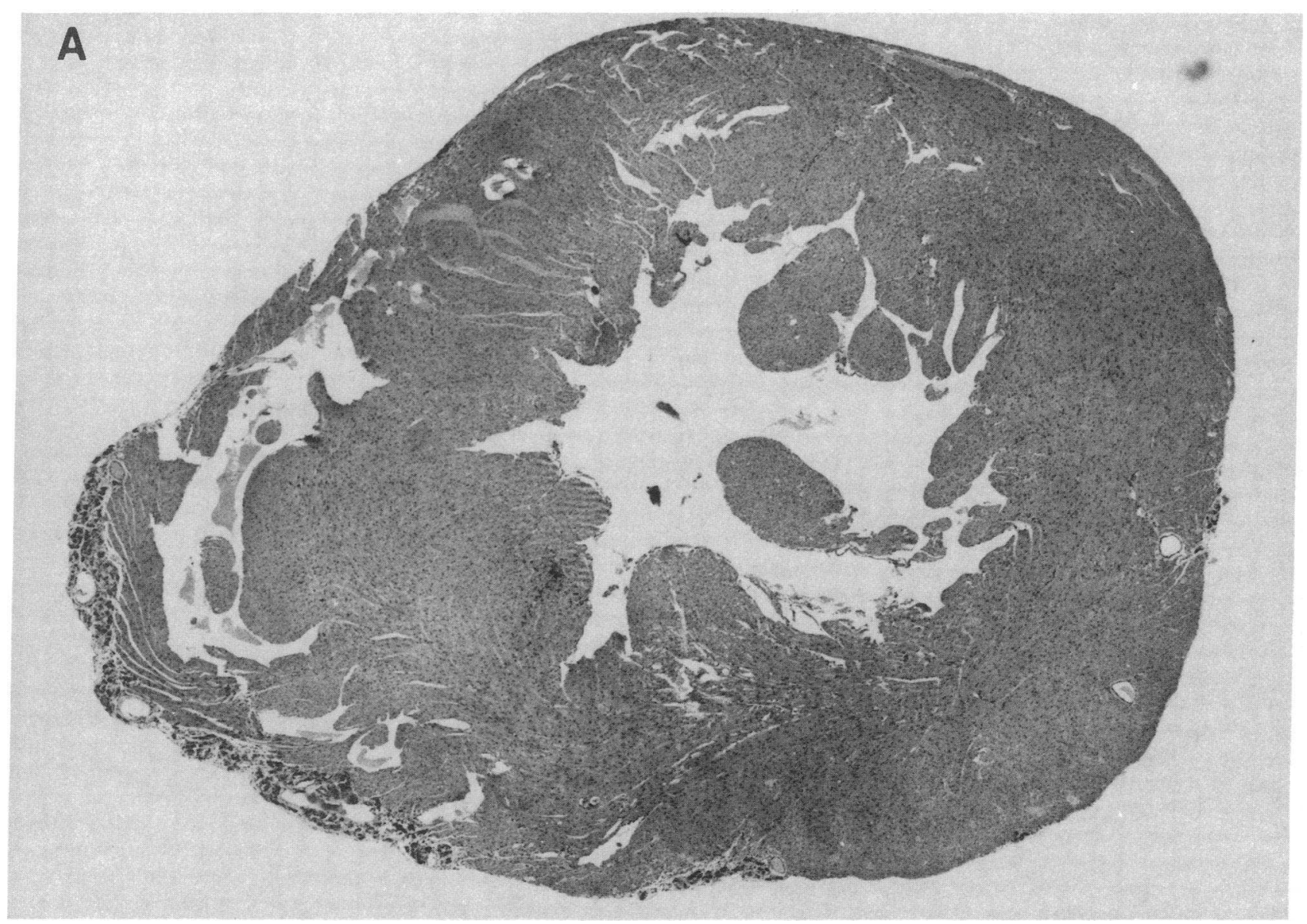

Figure 4. Light microscopic sections of representative uninfected control hearts. $(A)$ Low power $(\times 15)$ transverse section across a verapamiltreated uninfected heart from group 4. No inflammatory lesions were seen in the left ventricle. There were small epicardial lesions over the right ventricle, which can be seen in this model. $(B)$ Higher power $(\times 200)$ magnification of a section from group 5 (untreated uninfected control). The myocardial architecture was entirely normal.

sponse. Verapamil has been shown to selectively inhibit the expression and accumulation of IL-2 messenger RNA in mitogen-activated mononuclear cells (35).

Another aspect of the immune-mediated myocytolysis that may be attenuated by verapamil is the toxicity associated with autoantibodies found in myocarditis and dilated cardiomyopathy. An autoantibody to the ATP/ADP carrier has been found (35), and prolonged exposure of myocytes to this antibody has resulted in an enhanced calcium current and subsequent cell damage and death. This process was prevented by the addition of calcium channel blockers (36).

The pathologic picture of multiple focal areas of myocyte loss, fibrosis, and calcification diffusely distributed across all cardiac muscle layers and chambers characterizes several dilated cardiomyopathies of differing etiology. These pathological findings are exhibited by the cardiomyopathic Syrian hamster, a genetically transmitted model of myocardial dilatation and failure. Factor and co-workers (20) noted the fesemblance between the myocardial lesions of hamster cardiomyopathy and those observed after reperfusion injury and suggested that reperfusion injury at a microcirculatory level may be of pathogenetic importance for hamster heart disease. These investigators perfused normal and cardiomyopathic (before the pheno- typic expression of the disease) hamster hearts in vivo with liquid silicone rubber and were able to demonstrate that the onset of the cardiomyopathy was associated with numerous areas of microvascular constriction or spasm. These abnormalities did not represent fixed anatomic alterations of these microvessels but rather appeared to be potential sites of ischemia and reperfusion. This pathological and microvascular picture was subsequently seen in the hearts of rats with hypertension and diabetes (37), or after acute brain injury (38), and also in murine models of Chagasic cardiomyopathy (39).

Verapamil administration markedly ameliorates the microvascular disorder and the cardiomyopathy of both the Syrian hamster and the Chagasic mouse $(21,39-41)$. This benefit is postulated to be secondary to the prevention of free radical lipid peroxidation and myocyte injury after recurrent ischemia and reperfusion. In addition, verapamil may also reduce an imbalance of transsarcolemmal calcium flux and a toxic calcium overload of the myocyte (41). The microvascular spasm itself may be secondary to endothelial cell damage as a consequence of an affinity that the picornaviruses have for these cells.

We were not disappointed by the lack of differences in acute mortality in this study between the control and treated 


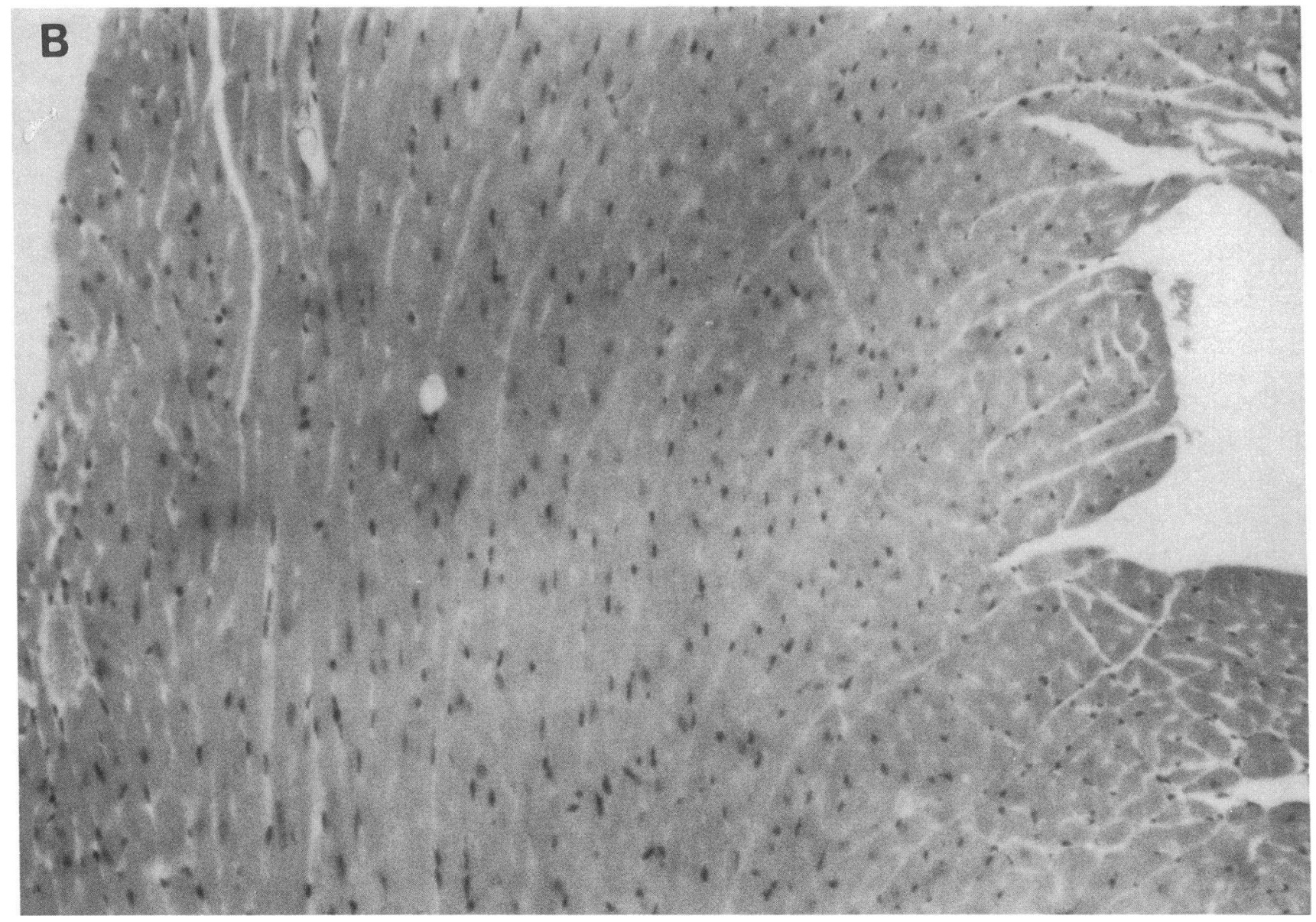

Figure 4 (Continued)

animals, but observed only a delay in mortality in the treated group. The data from group 1 and group 2 animals taken together suggested that the protective action of verapamil in this

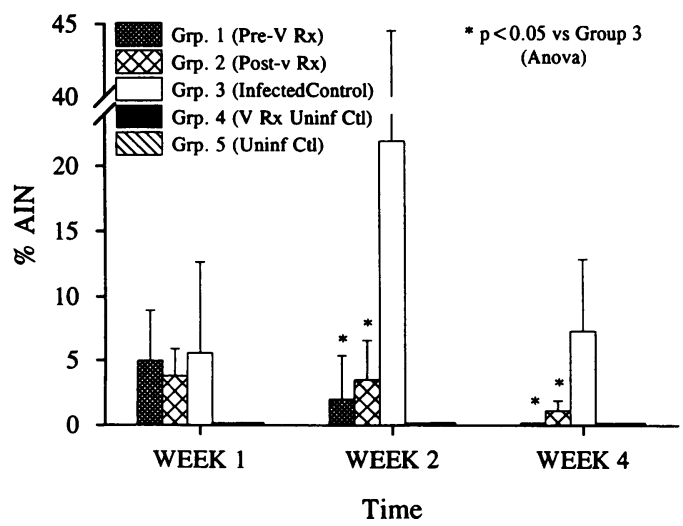

Figure 5. Percentage of planimetered absolute areas of inflammation and necrosis indexed to the total myocardial cross-sectional area. Verapamil-treated animals showed a significant decrease in areas of pathological involvement compared with infected controls. The uninfected animals showed no evidence of pathological involvement.

Data are expressed as group means $\pm \mathrm{SD}$. $A I N$, area of inflammation and necrosis; $V$, verapamil; $R x$, treatment; $C t l$, control; Uninf, uninfected; Anova, analysis of variance. model is during the late phase of the disease, interfering with the development of cardiomyopathy. This was confirmed by pathology, where the inflammation and necrosis during the first week was not dramatically different between the treated and untreated animals, but was significantly different after $14 \mathrm{~d}$ (peak infection). As the majority of animals die during the first and second weeks of the disease as the result of the acute infection, verapamil probably did not offer protection in this phase. Furthermore, there are many mechanisms accounting for the early mortality, including acute myocardial inflammation, diffuse toxic states, and extensive viral infections elsewhere, including the central nervous system, pancreas, and gastrointestinal tract. Indeed, inflammation by histological grading did not differ between the treated and control groups during week 1 of infection (Fig. 1), confirming that acute myocardial inflammation, which can lead to severe myocardial dysfunction and early mortality, was not affected by verapamil. Preliminary examination of the central nervous system also confirmed that the acute transverse myelitis seen in this model was not different between the control and treated groups in the early phases of the disease. We suspect that verapamil may have an impact on late mortality since it reduced the incidence of cardiomyopathy. However, this study was not designed to study late mortality as the primary end point, and will require a separate study with longer follow-up. Therefore, in this study we have demonstrated that the calcium channel blocker verapamil is capable 

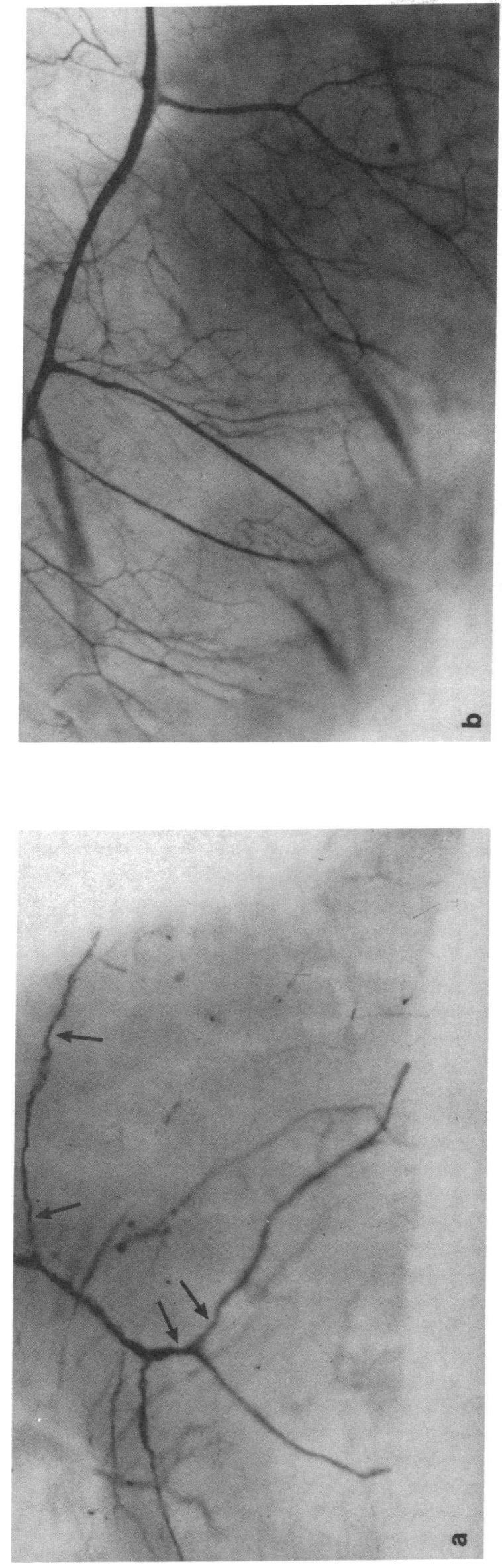

of attenuating the histological features of myocarditis even when given after viral inoculation. Until the mechanism of action of verapamil in myocarditis is fully understood, the specific therapy for myocarditis remains elusive. However, verapamil may provide directions toward new therapeutic approaches in myocarditis, and the potential of offering treatment for myocarditis even after virus infection has taken place. It also underscores the possible role of calcium overload and microvascular ischemia as a common motif in the generation of dilated cardiomyopathy from diverse etiologies.

In conclusion, the medical treatment of viral myocarditis has focused on the use of steroids and other immunosuppressive agents with little success. In this study we have confirmed that encephalomyocarditic viral myocarditis in the mouse is associated with spasm of the coronary microvasculature. Although the etiology of the microvascular abnormality is uncertain, it is clear that the immunological response to viral infection may sensitize both myocytes and cardiac vascular smooth muscle cells to calcium overload, resulting in spasm, myocyte injury, and necrosis. In light of these observations, verapamil administration to the infected animals may have significantly reduced the microvascular changes and attenuated the myocardial necrosis, fibrosis, and calcification characteristics of dilated cardiomyopathy. Verapamil treatment may be of benefit, therefore, at two levels: $(a)$ through inhibition of the immune response, and $(b)$ directly on the microvasculature. This also suggests a potential new therapeutic approach in treating clinical myocarditis. However, while EMC viral infection is an excellent model of viral myocarditis and subsequent cardiomyopathy, it rarely produces lesions in man. Hence, whether these observations are directly applicable in human cases of myocarditis and cardiomyopathy will need to be independently confirmed in the future.

\section{Acknowledgments}

We would like to thank Dr. Akira Matsumori of Kyoto University for the source of the EMC virus. We thank Dr. Campbell of the Virology Laboratory at the University of Toronto for the initial propagation of the viruses. We extend our appreciation to Knoll Pharmaceutical and Searle Canada for support and for the supply of verapamil in various preparations.

This work was supported by the Heart and Stroke Foundation of Ontario and the Medical Research Council of Canada.

\section{References}

1. Kereiakes, D. J., and W. W. Parmley. 1984. Myocarditis and cardiomyopathy. Am. Heart J. 108:1318-1326.

2. Kopecky, S. I., and B. J. Gersh. 1987. Dilated cardiomyopathy and myocarditis: natural history, etiology, clinical manifestations and management. Curr. Probl. Cardiol. 12:569-647.

3. Huber, S. A., and P. A. Lodge. 1984. Coxsackievirus B-3 myocarditis in Balb/c mice: evidence for autoimmunity to myocyte antigens. Am. J. Pathol. 116:21-29.

4. Huber, S. A., and P. A. Lodge. 1986. Coxsackievirus B3 myocarditis: identification of different pathogenic mechanisms in DBA/2 and Balb/c mice. Am. J. Pathol. 122:284-291.

5. Neu, N., K. W. Beisel, M. D. Traystman, N. R. Rose, and S. W. Craig. 1987. Autoantibodies specific for the cardiac myosin isoform are found in mice susceptible to Coxsackievirus B3-induced myocarditis. J. Immunol. 138:2488-2492.

6. Hosenpud, J. D., J. H. McAnulty, and N. R. Niles. 1985. Lack of objective improvement in ventricular systolic function in patients with myocarditis treated with azathioprine and prednisone. J. Am. Coll. Cardiol. 6:797-801.

7. Mason, J. W., M. E. Billingham, and D. R. Ricci. 1980. Treatment of acute inflammatory myocarditis assisted by endomyocardial biopsy. Am. J. Cardiol. 45:1037-1044. 
8. O’Connell, J. B., E. A. Reap, and J. A. Robinson. 1986. The effects of cyclosporine on acute murine Coxsackie B3 myocarditis. Circulation. 73:353359.

9. Kishimoto, C., K. A. Thorp, and W. H. Abelmann. 1990. Immunosuppression with high doses of cyclophosphamide reduces the severity of myocarditis but increases the mortality in murine Coxsackie B3 myocarditis. Circulation. 82:982-989.

10. Contanzo-Nordin, M. R., E. A. Reap, J. B. O'Connell, J. A. Robinson, and P. J. Scanlon. 1985. A nonsteroidal anti-inflammatory drug exacerbates Coxsackie B3 murine myocarditis. J. Am. Coll. Cardiol. 6:1078-1082.

11. Rezkalla, S., G. Khatib, and R. Khatib. 1986. Coxsackievirus B3 murine myocarditis: deleterious effects of nonsteroidal anti-inflammatory agents. $J$. Lab Clin. Med. 107:393-395.

12. Matsumori, A., and C. Kawai. 1982. An animal model of congestive (dilated) cardiomyopathy: Dilatation and hypertrophy of the heart in chronic stage in DBA/ 2 mice with myocarditis caused by encephalomyocarditis virus. Circulation. 66:355-360.

13. Chow, L. H., C. J. Gauntt, and B. M. McManus. 1991. Differential effects of myocarditic variants of coxsackievirus B3 in inbred mice: a pathologic characterization of heart tissue damage. Lab. Invest. 64:55-64.

14. Tomioka, N., C. Kishimoto, A. Matsumori, and C. Kawai. 1986. Effects of prednisolone on acute viral myocarditis in mice. J. Am. Coll. Cardiol. 7:868872 .

15. Monrad, E. S., A. Matsumori, J. C. Murphy, J. G. Fox, C. S. Crumpacker, and W. H. Abelmann. 1986. Therapy with cyclosporine in experimental murine myocarditis with encephalomyocarditis virus. Circulation. 73:1058-1064.

16. Bowles, N. E., P. J. Richardson, E. G. J. Olsen, and L. C. Archard. 1986 Detection of Coxsakie B virus specific RNA sequences in myocardial biopsy samples from patients with myocarditis and dilated cardiomyopathy. Lancet. i:1120-1123.

17. Jin, O., M. J. Sole, J. W. Butany, W. K. Chia, P. R. McLaughlin, P. Liu, and C. C. Liew. 1990. The detection of enterovirus RNA in myocardial biopsies from patients with myocarditis and cardiomyopathy using gene amplification by the polymerase chain reaction. Circulation. 82:8-16.

18. Huber, S. A., L. P. Job, and J. F. Woodruff. 1980. Lysis of infected myofibers by Coxsackie B3-immune T lymphocytes. Am. J. Pathol. 98:681-694.

19. Silver, M. A., and D. Kowalczyk. 1989. Coronary microvascular narrowing in acute murine coxsackie B3 myocarditis. Am. Heart J. 118:173-174.

20. Factor, S. M., T. Minase, S. Cho, R. Dominitz, and E. H. Sonnenblick. 1982. Microvascular spasm in the cardiomyopathic Syrian hamster: a prevental cause of focal myocardial necrosis. Circulation. 66:342-354.

21. Morris, S. A., L. M. Weiss, S. M. Factor, J. P. Bilizekian, H. Tanowitz, and M. Witiner. 1989. Verapmil ameliorates clinical, pathologic and biochemical manifestations of experimental chagasic cardiomyopathy in mice. J. Am. Coll. Cardiol. 14:782-789.

22. Chow, L. H., K. W. Beisel, and B. M. McManus. 1992. Enteroviral infection of mice with severe combined immunodeficiency: evidence for direct viral pathogenesis of myocardial injury. Lab. Invest. 66:24-31.

23. Kishimoto, C., K. Kuribayashi, K. Fukuma, T. Masuda, N. Tomioka W. H. Abelmann, and C. Kawai. 1987. Immunologic identification of lympho cyte subsets in experimental murine myocarditis with encephalomyocarditis virus. Circ. Res. 61:715-725.

24. Kishimoto, C., K. Kuribayashi, T. Masuda, N. Tomioka, and C. Kawai. 1985. Immunological behaviour of lymphocytes in experimental viral myocarditis: significance of $\mathrm{T}$ lymphocytes in the severity of myocarditis and silent myocarditis in BALB/c-nu/nu mice. Circulation. 71:1247-1254.
25. Kandolf, R., D. Ameis, P. Kirschener, A. Canu, and P. H. Hofschneider. 1987. In situ detection of enteroviral genomes in myocardial cells by nucleic acid hybridization: an approach to the diagnosis of viral heart disease. Proc. Natl. Acad. Sci. USA. 84:6272-6276.

26. Tracy, S., V. Wiegand, B. McManus, C. Gauntt, M. Pallansch, M. Beck, and N. Chapman. 1990. Molecular approaches to enteroviral diagnosis in idiopathic cardiomyopathy and myocarditis. J. Am. Coll. Cardiol. 15:1688-1694.

27. Schlesinger, M. J., and D. Cahill. 1989. Verapamil and chlorpromazine inhibit the budding of sindbis and vescular stomatitis viruses from infected chicken embryo fibroblasts. Virology. 168:187-190.

28. McManus, B. M., L. H. Chow, C. J. Gauntt, K. Klingel, and R. Kandolf. 1990. Relationship of early replicative intensity to persistence of enterovirus in murine myocarditis. Circulation. 82:III-290.

29. Hashimoto, I., and T. Komatsu. 1978. Myocardial changes after infection with Coxsackievirus B3 in nude mice. Br. J. Exp. Pathol. 59:13-20.

30. Hassin, D., R. Fixler, A. S. Kelin, H. Bank, and Y. Hassin. 1985. Cytotoxic $\mathrm{T}$ lymphocytes and natural killer cell activity in the course of mengovirus infection of mice. Immunology. 56:701-705.

31. Mills, G. B., R. K. Cheung, S. Grinstein, and E. W. Gelfand. 1985. Increase in cytosolic free calcium concentration is an intracellular messenger for the production of interleukin- 2 but not for expression of the interleukin- 2 receptor. J. Immunol. 134:1640-1643.

32. Gelfand, E. W., R. K. Cheung, S. Grinstein, and G. B. Mills. 1986. Characterization of the role for calcium influx in mitogen-induced triggering of human $T$ cells: identification of calcium-dependent and calcium independent signals. Eur. J. Immunol. 16:907-912.

33. Birx, D. L., M. Berger, and T. A. Fleisher. 1984. The interference of T cell activation by calcium channel blocking agents. J. Immunol. 133:2904-2909.

34. Wright, B., I. Zeidman, R. Greig, and G. Psote. 1985. Inhibition of macrophage activation by calcium channel blockers and calmodulin antagonists. Cell. Immunology. 95:46-53.

35. Schultheiss, H. P., K. Schulze, U. Kuhl, G. Ulrich, and M. Klingenberg. 1987. The ADP/ATP carrier as a mitochondrial autoantigen: facts and perspectives. Ann. NY Acad. Sci. 488:44-56.

36. Schulze, K., B. Becker, and H. P. Schultheiss. 1989. Antibodies to the ADP/ATP carrier, an autoantigen in myocarditis and dilated cardiomyopathy, penetrate into myocardial cells and disturb energy metabolism in vivo. Circ. Res. 64:179-192.

37. Factor, S. M., T. Minase, S. Cho, F. Fein, J. M. Capasso, and E. H. Sonnenblick. 1984. Coronary microvascular abnormalities in the hypertensivediabetic rat: a primary cause of cardiomyopathy? Am. J. Pathol. 116:9-20.

38. Shanlin, R. J., M. J. Sole, M. Rahimifar, C. H. Tator, and S. M. Factor. 1988. Increased intracranial pressure elicits hypertension, increased sympathetic activity, electrocardiographic abnormalities and myocardial damage in rats. $J$. Am. Coll. Cardiol. 12:727-736.

39. Factor, S. M., S. Cho, M. Witiner, and H. Tanowitz. 1985. Abnormalities of the coronary microcirculation in acute murine Chagas' disease. Am. J. Trop. Med. Hyg. 1985:246-253.

40. Kobayashi, A., T. Yamashita, M. Kaneko, T. Nishiyama, M. Mayashi, and N. Yamazaki. 1987. Effect of verapamil on experimental cardiomyopathy in the Bio 14.6 Syrian hamster. J. Am. Coll. Cardiol. 10:1128-1134.

41. Rouleau, J. L., L. H. S. Chuck, G. Hollosi, P. Kidd, R. E. Sievers, J. Wikman-Coflett, and W. W. Parmley. 1982. Verapamil preserves myocardial contractility in the hereditary cardiomyopathy of the Syrian hamster. Circ. Res. $50: 405-412$ 